\title{
ON THE COMPUTATION OF INFELD'S FUNCTION USED IN EVALUATING THE ADMITTANCE OF PROLATE SPHEROIDAL DIPOLE ANTENNAS
}

$\mathrm{BY}$

\author{
T. DO-NHAT AND R. H. MACPHIE
}

Dept. of Electrical and Computer Engineering, University of Waterloo, Canada

\begin{abstract}
The Infeld function expressed in terms of the outgoing prolate spheroidal radial wave function and its derivative, and employed in the expression of the input self-admittance of prolate spheroidal antennas, has accurately been calculated by using a newly developed asymptotic expression for large degree $n$. This asymptotic power series has been derived by using a perturbation method with a perturbation parameter $\epsilon=1 /\left(\lambda_{1 n}-h^{2}\right)$, where $\lambda_{1 n}$ is the spheroid's eigenvalue for the given parameter $h$ of the spheroidal wave function.
\end{abstract}

1. Introduction. In the computation of the admittance of prolate spheroidal dipole antennas of finite feed gap length [1], [2], [3] we encounter the problem of the slow convergence of the input susceptance, expressed as an infinite sum of outgoing prolate spheroidal wave functions, and their first-order derivative. More specifically, each term of the susceptance includes the following function:

$$
r_{n}(h, \xi)=\frac{\left(\xi^{2}-1\right)^{1 / 2} R_{1 n}^{(4)}(h, \xi)}{\frac{d}{d \xi}\left[\left(\xi^{2}-1\right)^{1 / 2} R_{1 n}^{(4)}(h, \xi)\right]}
$$

Infeld $\left[1\right.$, p. 126] chose $\zeta_{n-1}$ to represent this function. In $(1) R_{1 n}^{(4)}(h, \xi)$ is the prolate spheroidal radial function of order 1 and degree $n$ of the fourth kind [4]. It satisfies the following differential equation [4]:

$$
\frac{d}{d \xi}\left\{\left(\xi^{2}-1\right) \frac{d}{d \xi} R_{1 n}^{(4)}(h, \xi)\right\}-\left(\lambda_{1 n}-h^{2} \xi^{2}+\frac{1}{\xi^{2}-1}\right) R_{1 n}^{(4)}(h, \xi)=0, \quad n=1,2,3,4, \ldots
$$

where $\xi$ is the spheroid's radial coordinate $(\xi \geq 1)$, and $\lambda_{1 n}$ is the spheroid's eigenvalue for the given $h$ parameter, i.e., $h=k F$ with $k$ being the operating wavenumber and $F$ the semi-interfocal distance of the spheroid.

Received July 26, 1994.

This work was supported by the Natural Sciences and Engineering Research Council, Ottawa, Grant A2176. 
The Infeld function $r_{n}(h, \xi)$ can be computed from $R_{1 n}^{(4)}(h, \xi)$ and its first-order derivative, which have recently been calculated with single [5] and double [6] precision accuracies. However, for large degree $n$ it is useful to develop an asymptotic expression of $r_{n}(h, \xi)$. Infeld [1, p. 126] gave only the leading term of the function.

By introducing a perturbation parameter into the Riccati differential equation that the Infeld function satisfies, and by using the perturbation theory [7], its remaining asymptotic terms have been found. This asymptotic expression has been proved to be helpful in obtaining a high accuracy for large $n$.

2. Asymptotic expression of the Infeld function. First, by letting

$$
P_{n}(h, \xi)=\left(\xi^{2}-1\right)^{1 / 2} R_{1 n}^{(4)}(h, \xi),
$$

we obtain from $(2)$

$$
\left(\xi^{2}-1\right) \frac{d^{2}}{d \xi^{2}} P_{n}(h, \xi)-\left(\lambda_{1 n}-h^{2} \xi^{2}\right) P_{n}(h, \xi)=0 .
$$

Because of (4), the Infeld function given by (1) satisfies the following Riccati differential equation:

$$
\frac{d}{d \xi} r_{n}(h, \xi)+r_{n}^{2}(h, \xi)\left[\frac{\lambda_{1 n}}{\xi^{2}-1}-\frac{h^{2} \xi^{2}}{\xi^{2}-1}\right]=1 .
$$

Equation (5) was also derived by Infeld.

Now, if we introduce the perturbation parameter

$$
\epsilon=\frac{1}{\lambda_{1 n}-h^{2}}
$$

and use the following transformation:

$$
s_{n}(h, x)=\left(\frac{\lambda_{1 n}-h^{2}}{\xi^{2}-1}\right)^{1 / 2} r_{n}(h, \xi), \quad x=\frac{\sqrt{\xi^{2}-1}}{\xi},
$$

(5) is reduced to

$$
\sqrt{\epsilon} x\left(1-x^{2}\right) \frac{d s_{n}(h, x)}{d x}+\sqrt{\epsilon} s_{n}(h, x)+x s_{n}^{2}(h, x)\left(1-h^{2} \frac{x^{2}}{1-x^{2}} \epsilon\right)-x=0 .
$$

In (8) if $\epsilon \rightarrow 0$ we obtain $s_{n}^{2}(h, x)=1$ and choose $s_{n}(h, x)=-1$ to have a physical meaning [1]. This is also the only leading term obtained by Infeld.

Therefore, by employing a perturbation theory [7], the function $s_{n}(h, x)$ can be assumed to be expanded in rational powers of $\epsilon$ with the weighting functions $h_{m}(h, x)$ to be found, as follows:

$$
s_{n}(h, x)=\sum_{m=0}^{\infty} \epsilon^{m / 2} h_{m}(h, x)
$$

The above asymptotic expansion was extensively discussed by Kevorkian and Cole [7]. 
The substitution of (9) into (8) yields

$$
\begin{aligned}
& \sum_{m=0}^{\infty}\left[x\left(1-x^{2}\right) \frac{d h_{m}(h, x)}{d x}+h_{m}(h, x)\right] \epsilon^{(m+1) / 2} \\
& \quad+x\left[\sum_{m=0}^{\infty} \sum_{l=0}^{m} h_{l}(h, x) h_{m-l}(h, x)\left(1-\frac{h^{2} x^{2}}{1-x^{2}} \epsilon\right) \epsilon^{m / 2}-1\right]=0 .
\end{aligned}
$$

By comparing like rational powers of $\epsilon$ in (10) we find the relationship among the weighting functions $h_{m}(h, x)$ as follows:

$$
\begin{gathered}
h_{0}^{2}(h, x)=1, \\
2 x h_{m+1}(h, x)=x\left(1-x^{2}\right) \frac{d h_{m}(h, x)}{d x}+h_{m}(h, x)+\sum_{l=1}^{m} x h_{l}(h, x) h_{m+1-l}(h, x) \\
-h^{2} \frac{x^{3}}{1-x^{2}} \sum_{l=0}^{m-1} h_{l}(h, x) h_{m-1-l}(h, x), \quad m=1,2,3, \ldots
\end{gathered}
$$

The recursion relation (12) shows that we can find $h_{m+1}(h, x)$ provided that the weighting functions $h_{k}(h, x)$ with $k=0,1,2, \ldots, m$ are known. From Eq. (11) we obtain two solutions for $h_{0}(h, x)$, of which only one solution has a physical meaning, i.e., $h_{0}(h, x)=$ -1 , which is Infeld's only term. If we commence with his term, by using (12) the closed forms of $h_{1}(h, x), h_{2}(h, x), \ldots, h_{6}(h, x)$ are given by

$$
\begin{gathered}
h_{1}(h, x)=-\frac{1}{2 x}, \quad h_{2}(h, x)=-\frac{1}{4}+\frac{1}{8 x^{2}}-\frac{h^{2}}{2} \frac{x^{2}}{1-x^{2}}, \\
h_{3}(h, x)=-\frac{1}{8 x^{3}}\left(1-x^{2}\right)-h^{2} \frac{x}{1-x^{2}}, \\
h_{4}(h, x)=\frac{25}{128} \frac{1}{x^{4}}-\frac{9}{32 x^{2}}+\frac{3}{32}+\frac{h^{2}}{8} \frac{1}{1-x^{2}}\left(-\frac{9}{2}-5 x^{2}-3 h^{2} \frac{x^{4}}{1-x^{2}}\right), \\
h_{5}(h, x)=-\frac{13}{32} \frac{1}{x^{5}}+\frac{23}{32} \frac{1}{x^{3}}-\frac{5}{16} \frac{1}{x}-\frac{5}{8} h^{2} x-h^{2} \frac{x}{1-x^{2}}\left(\frac{3}{8}+\frac{1}{8 x^{2}}+\frac{5}{8} x^{2}+\frac{3}{2} \frac{h^{2} x^{2}}{1-x^{2}}\right), \\
h_{6}(h, x)=\frac{1073}{1024} \frac{1}{x^{6}}-\frac{1123}{512} \frac{1}{x^{4}}+\left(\frac{339}{256}+\frac{h^{2}}{8}\right) \frac{1}{x^{2}}-\left(\frac{23}{128}+\frac{h^{2}}{2}\right)-\frac{5}{8} h^{2} x^{2} \\
+\frac{h^{2}}{1-x^{2}}\left(\frac{5}{256} \frac{1}{x^{2}}-\frac{11}{64}-\frac{13}{64} x^{2}-\frac{5}{8} x^{4}\right) \\
-h^{4}\left(1-x^{2}\right)^{2} \frac{x^{2}}{64}\left(70 x^{2}+161\right)-\frac{5}{16} \frac{h^{6} x^{6}}{\left(1-x^{2}\right)^{3}} .
\end{gathered}
$$

The generation of higher-order weighting functions $h_{m}(h, x)$ is more tedious. However, in the appendix it is shown that there also exists a recursion relation among the expansion 
coefficients of $h_{m}(h, x)$, which facilitates their systematic computation. Therefore, the final form of the Infeld function, with the use of Eqs. (6), (7), and (9), is given by

$$
r_{n}(h, \xi)=\sqrt{\xi^{2}-1} \sum_{m=0}^{\infty}\left(\lambda_{1 n}-h^{2}\right)^{-(m+1) / 2} h_{m}(h, x), \quad x=\sqrt{\xi^{2}-1} / \xi .
$$

Equation (14) has been derived under the asymptotic condition, i.e., $x \sqrt{\lambda_{1 n}-h^{2}}>1$.

3. Numerical results. Table 1 shows the convergence characteristics of the asymptotic series of (14) in terms of its number of terms $N$ for $\xi=1.05, h=2$ and $n=15,17$, and 19. The computed values are verified with those calculated with a double precision accuracy [6]. It is shown from the table that we have obtained about 5 to 6 significant figures of accuracy with only 7 terms of the asymptotic series for $x \sqrt{\lambda_{1 n}-h^{2}}>3$. Better results can be obtained by increasing $n$ or $N$. For thin spheroids, i.e., $\xi=1.005$, Table 2 gives the computed values of $r_{n}(h, \xi)$ with 7 terms for $n=50,51, \ldots, 63$ to ensure about six significant figures of accuracy.

TABLE 1. Convergence characteristics of the asymptotic series of (14) in terms of its number of terms $N$ for $\xi=1.05$ and $h=2$ for large degree $n$. The true values [6] of $r_{n}(h, \xi)$ are $-2.290737 \times 10^{-2}$, $-2.005182 \times 10^{-2},-1.782769 \times 10^{-2}$ for $n=15,17,19$, respectively.

\begin{tabular}{|c|c|c|c|}
\hline$N$ & $n=15$ & $n=17$ & $n=19$ \\
\hline 1 & $-2.07528 \times 10^{-2}$ & $-1.83623 \times 10^{-2}$ & $-1.64671 \times 10^{-2}$ \\
2 & $-2.29588 \times 10^{-2}$ & $-2.00893 \times 10^{-2}$ & $-1.78560 \times 10^{-2}$ \\
3 & $-2.28812 \times 10^{-2}$ & $-2.00356 \times 10^{-2}$ & $-1.78173 \times 10^{-2}$ \\
4 & $-2.29114 \times 10^{-2}$ & $-2.00541 \times 10^{-2}$ & $-1.78292 \times 10^{-2}$ \\
5 & $-2.29052 \times 10^{-2}$ & $-2.00507 \times 10^{-2}$ & $-1.78273 \times 10^{-2}$ \\
6 & $-2.29084 \times 10^{-2}$ & $-2.00523 \times 10^{-2}$ & $-1.78281 \times 10^{-2}$ \\
7 & $-2.29067 \times 10^{-2}$ & $-2.00516 \times 10^{-2}$ & $-1.78278 \times 10^{-2}$ \\
\hline
\end{tabular}

TABLE 2. Values of the Infeld function $r_{n}(h, \xi)$ computed from (14) using 7 terms for $\xi=1.005$ and $h=2$ with large $n(n=50,51, \ldots, 63)$

\begin{tabular}{|c|c|c|c|}
\hline$n$ & $r_{n}(h, \xi)$ & $n$ & $r_{n}(h, \xi)$ \\
\hline 51 & $-2.12694 \times 10^{-3}$ & 50 & $-2.17263 \times 10^{-3}$ \\
53 & $-2.04104 \times 10^{-3}$ & 52 & $-2.08311 \times 10^{-3}$ \\
55 & $-1.96177 \times 10^{-3}$ & 54 & $-2.00063 \times 10^{-3}$ \\
57 & $-1.88839 \times 10^{-3}$ & 56 & $-1.92439 \times 10^{-3}$ \\
59 & $-1.82027 \times 10^{-3}$ & 58 & $-1.85371 \times 10^{-3}$ \\
61 & $-1.75686 \times 10^{-3}$ & 60 & $-1.78801 \times 10^{-3}$ \\
63 & $-1.69770 \times 10^{-3}$ & 62 & $-1.72678 \times 10^{-3}$ \\
\hline
\end{tabular}


Appendix. From Eqs. (11), (12), and (13) the weighting function $h_{m}(h, x)$ is expanded as follows:

$$
h_{m}(h, x)=x^{-m} \sum_{k=0}^{\infty} a_{m k}(h) x^{2 k},
$$

with its derivative given by

$$
\frac{d h_{m}(h, x)}{d x}=\sum_{k=0}^{\infty}(2 k-m) a_{m k}(h) x^{2 k-m-1} .
$$

The substitution of (A1) and (A2) into the recursion relation (12) yields after some algebra:

$$
\begin{aligned}
2 \sum_{k=0}^{\infty} a_{m+1, k}(h) x^{2 k-m}= & \sum_{k=0}^{\infty}(2 k-m) a_{m k}(h) x^{2 k-m}-\sum_{k=0}^{\infty}(2 k-n) a_{m k}(h) x^{2 k-m+2} \\
& +\sum_{k=0}^{\infty} a_{m k}(h) x^{2 k-m}+\sum_{s=0}^{\infty} \sum_{k=0}^{s} \sum_{l=1}^{m} a_{l k}(h) a_{m+1-l, s-k}(h) x^{2 s-m} \\
& -h^{2} \sum_{t=0}^{\infty} \sum_{s=0}^{t} \sum_{k=0}^{s} \sum_{l=0}^{m-1} a_{l k}(h) a_{m-1-l, s-k}(h) x^{2 t-m+4}
\end{aligned}
$$

By comparing like powers of $x$, the recursion relation among the expansion coefficients $a_{m l}(h)$ is obtained as follows:

$$
\begin{aligned}
a_{m+1, l}(h)= & \frac{1}{2}(2 l-m+1) a_{m l}(h)-\frac{1}{2}(2 l-m-2) a_{m, l-1}(h) \\
& +\frac{1}{2} \sum_{k=0}^{l} \sum_{r=1}^{m} a_{r k}(h) a_{m+1-r, l-k}(h) \\
& -\frac{h^{2}}{2} \sum_{r=0}^{l-2} \sum_{k=0}^{r} \sum_{s=0}^{m-1} a_{s k}(h) a_{m-1-s, r-k}(h) .
\end{aligned}
$$

If we begin with the expansion coefficient of $h_{0}(h, x)$, i.e., $a_{0, l}(h)=-\delta_{0, l}$, the coefficients $a_{m l}(h)(m=1,2, \ldots ; l=0,1,2, \ldots)$ of $h_{m}(h, x)$ can easily be generated from (A4).

\section{REFERENCES}

[1] L. Infeld, The influence of the width of the gap upon the theory of antennas, Quart. Appl. Math. 5, 113-132 (1947)

[2] J. D. Kotulski, Transient radiation from antennas: Early time response of the spherical antenna and the late time response of the prolate spheroidal impedance antenna, Univ. of Illinois at Chicago, Illinois, Ph. D. Dissertation, 1983

[3] T. Do-Nhat and R. H. MacPhie, The input admittance of thin prolate spheroidal dipole antennas with finite gap widths, IEEE Trans. AP-43, 1995, pp. 1243-1252

[4] C. Flammer, Spheroidal Wave Functions, Stanford University Press, Stanford, Calif., 1957

[5] B. P. Sinha and R. H. MacPhie, On the computation of the prolate spheroidal radial functions of the second kind, J. Math. Phys. 16, 2378-2381 (1975)

[6] T. Do-Nhat and R. H. MacPhie, On the accurate computation of the prolate spheroidal radial functions of the second kind, Quart. Appl. Math. 54, 677-685 (1996)

[7] J. Kevorkian and J. D. Cole, Perturbation Methods in Applied Mathematics, Springer-Verlag, New York, 1980 